\title{
Model Based Design of a Split Carrier Wheel Suspension for Light-weight Vehicles
}

\author{
Jakub Tobolár ${ }^{1} \quad$ Daniel Baumgartner ${ }^{1} \quad$ Yutaka Hirano $^{2} \quad$ Tilman Bünte $^{1} \quad$ Michael Fleps-Dezasse $^{1}$ \\ Jonathan Brembeck ${ }^{1}$ \\ ${ }^{1}$ German Aerospace Center (DLR), Institute of System Dynamics and Control, Wessling, \\ \{Daniel.Baumgartner, Jakub.Tobolar\} @DLR.de \\ ${ }^{2}$ Toyota Motor Corporation, Future Project Division, Shizuoka, Japan, \\ Yutaka_Hirano@mail.toyota.co.jp
}

\begin{abstract}
Applying light-weight construction methods to the design of future electric vehicles results in weight reduction of both the vehicle body and the chassis. However, the potential for percental reduction of the sprung mass is larger compared to that of the unsprung mass. Consequently, unfavorable consequences on the compromise, which always needs to be found between road contact and road holding, can arise. This requires additional arrangements in order to reach the performance of a state-of-the-art conventional vehicle. This paper presents a possible design solution. The wheel carrier is split into two parts, thus enabling to tune the frequency response correspondingly to reference vehicles. Besides the technical solution the Modelica modeling of the proposed suspension system as well as a vehicle dynamics and ride comfort assessment are presented.
\end{abstract}

Keywords: split wheel carrier, vehicle suspension, unsprung mass, small electric vehicle, three mass system

\section{Introduction}

Recently, to contribute to lower carbon dioxide emissions, the development of light-weight electric vehicles (LEV) has become more and more active in the automotive sector. These LEVs usually consume less energy in comparison to conventional vehicles. Though, because the sprung mass of those vehicles tends to be reduced relatively more than the unsprung mass of the suspension, the ratio of sprung mass to unsprung mass is directed toward lower values compared to those of conventional vehicles. This means that the resonance frequency of the sprung mass becomes closer to the resonance frequency of the unsprung mass which results in possible reduction of the ride comfort.

Furthermore, the resonance peaks associated with the sprung mass and the unsprung one are shifted to higher frequencies compared to those of conventional vehicles because of reduced masses of both parts. These phenomena arises when the resonance frequency of the unsprung mass becomes close to the drive shaft twisting mode. Thus, possibly a resonance excitation of the unsprung mass and the drive train can occur induced by adverse propelling torques.

To prevent abovementioned problems, it is necessary to shift the resonance frequency of the unsprung mass to lower / higher values and also to reduce the magnitude peak of the vertical acceleration frequency response associated with the unsprung mass. However, as shortly discussed in the next section, it is theoretically proven that the resonance frequency and the magnitude peak of the unsprung mass cannot be influenced by adjusting spring and damper coefficients of the conventional suspension which constitutes a two mass system of the unsprung mass and the sprung one.

To solve this design conflict, the idea of a three mass suspension system is introduced. In Section 2 of this paper, theoretical analysis of the effect of a three mass suspension is analyzed at first. A technical solution of the mechanical structure of the three mass system is described in Section 3. Actual effects of the three mass system are finally investigated by simulations using a Modelica model (presented in Section 4) of the proposed suspension together with an advanced multi-body vehicle model in Section 5. Section 6 provides a conclusion of the investigations results.

\section{Basic Idea of the Three Mass Sus- pension System}

Figure 1 (left) shows a schematic quarter car of a conventional "two mass suspension system". Here, $m_{b}$ denotes the mass of the sprung mass (representing a quarter of the car body) and $m_{w}$ is the mass of the unsprung mass composed of the wheel and its carrier block. Figure 2 shows a set of Bode magnitude plots from the acceleration of road excitation to the sprung mass acceleration of this two mass system. The parameter varied between the set of plots is the suspension spring stiffness $c_{b w}$ in the upper chart and damping $d_{b w}$ in the lower one. It can be concluded that the resonance peak associated with the sprung mass mode (second peak in the gain plot) can be changed neither by varying the spring stiffness, nor by variation of the damp- 

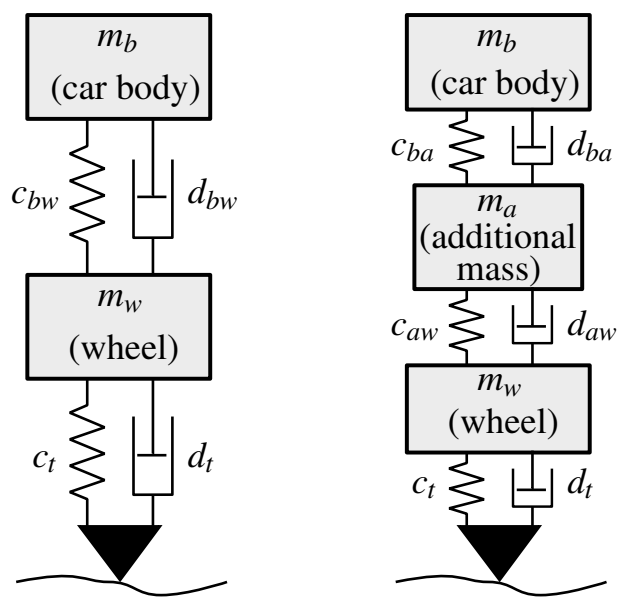

Figure 1. Schematic quarter car models of a conventional two mass suspension system (left) and of the three mass system (right).

ing of the suspension. This corresponds to the fundamental investigations of invariance properties of a two mass suspension system presented e.g. in (Hedrick and Butsuen, 1990) or (Savaresi et al., 2010), which emphasize that the sprung mass acceleration near to the wheel resonance frequency is invariant regarding body spring stiffness $c_{b w}$ and body damping $d_{b w}$. To overcome this fundamental restriction, the serial three mass suspension system as shown in Figure 1 (right) is introduced. In this solution, the unsprung mass of the wheel carrier is divided into two parts being insulated by an additional spring and damping elements ( $c_{a w}$ and $d_{a w}$, respectively) from each other. A detailed theoretical comparison of three mass systems with classical two mass systems highlighting the advantages of three mass systems is given in (Ryba, 1974a) and (Ryba, 1974b).

Figure 3 shows a Bode magnitude plot comparison of the two mass system with a set of plots for the three mass system when changing the spring stiffness $\left(c_{a w}\right)$ of the additional spring element. Here, the spring stiffness of the additional spring was set relative to the vertical tire stiffness $c_{t}$ using a factor $k$ as

$$
c_{a w}=k \cdot c_{t}
$$

It is shown by Figure 3 that by selecting a proper value of the gain coefficient $k$ such as $k=1.0$, it is possible to shift the frequency of the mode related to the unsprung mass and also to reduce the magnitude of the resonance peak. After this encouraging preliminary result it was decided to design a mechanic realization of such a three mass suspension with split carrier which will be presented and discussed in the following section.

\section{Technical Solution}

The possible technical solution of the three mass system was developed assuming a small LEV with state-of-the-art double wishbone front and rear suspensions.
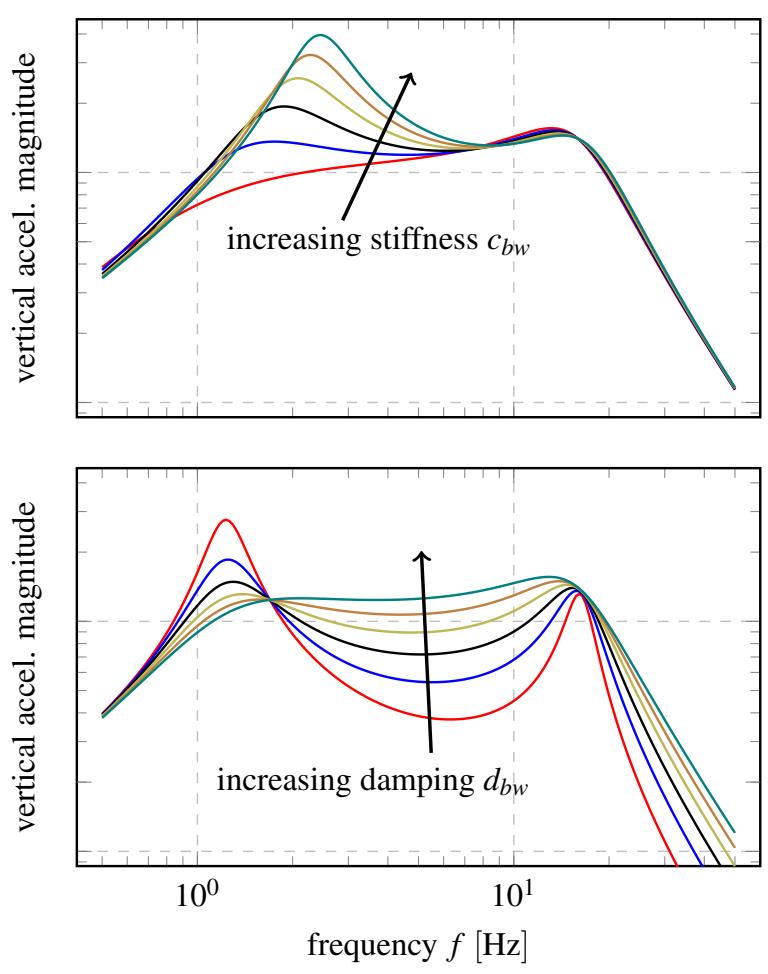

Figure 2. Bode magnitude plots from the acceleration of road excitation to the sprung mass acceleration of the two mass suspension system with varying spring stiffness (above) and varying damping (below).

The solution described below consists in splitting the wheel carrier into two parts - the wheel hub and the carrier itself - guided and suspended to each other. Thus, the lower part of the unsprung part $\left(m_{w}\right)$ consists of a wheel, a tire, a brake disc and a carrier which supports the wheel hub bearing and the brake caliper. The upper part of the split unsprung mass $\left(m_{a}\right)$ incorporates parts connecting suspension linkage mounts and the rest of the hub carrier. A mechanism to limit the relative motion between $m_{w}$ and $m_{a}$ has also to be considered. An additional spring element $\left(c_{a w}\right)$ is assumed as a rubber bushing element or combination of a bushing element and a supplementary spring element.

Several technical solutions were examined to realize abovementioned arrangement. Finally, the solution with linear sliding mechanism, see Figure 4, was chosen. It consists of two sealed linear sliding bearings (light grey parts in Figure 4) connected firmly with the wheel hub (purple) and guided through the supporting wheel carrier structure (beige). This design resembles a mechanism of a conventional telescopic fork as utilized for motorcycle front suspension; see (Stoffregen, 2012). The advantages are a simple, sealed and long-time proven design and availability of standardized parts thus enabling relatively cheap production and assembly. Moreover, such a design offers high stiffness against external forces and torques. Consequently, the wheel attitude changes under common 


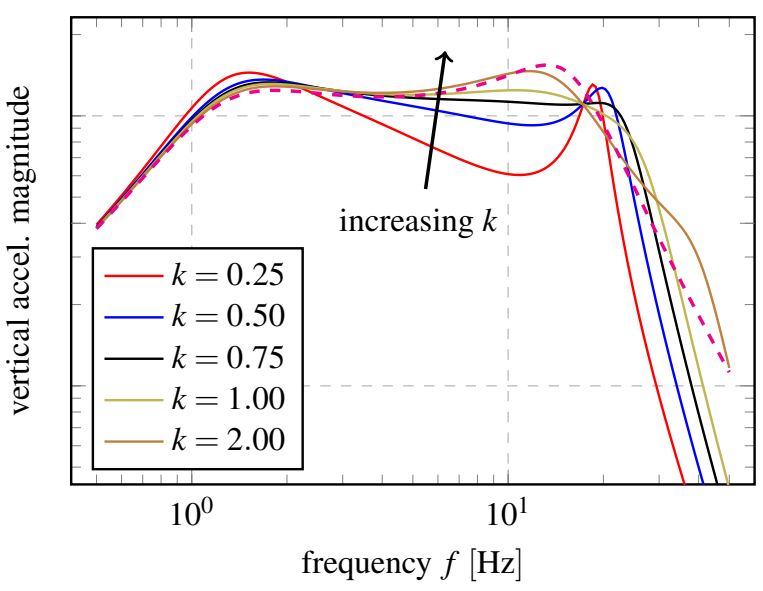

Figure 3. Bode magnitude plots of the three mass suspension system (using varying factor $k$ according to equation (1)) compared to the conventional two mass suspension (marked with dashed magenta line).

operating loads can be minimized, as proven by vehicle dynamic tests, see Section 5.3. For the investigated LEV, this technical solution was applied for both the front and the rear suspension, the latter being of the driven axle.

The wheel carrier support structure is depicted in more detail in Figure 5. It consists of support tubes with integrated sliding bearings (part D in Figure 5, yellow) and a rubber sealing (part E, green/black/orange). The wheel hub (purple) together with the brake's caliper (not depicted in Figure 5) is attached to immersion tubes in order to provide vertical deflection only between the parts of the wheel carrier.

The vertical motion of the split wheel carrier is suspended and damped by a rubber bushing element (part A in Figure 5, dark grey) together with support coil springs (part C, red/orange) housed within one of the tubes (light grey). The load springs are supported by threaded head caps (part F, light red) for easy exchange and adjustment of the spring preload. A non-linear damping device (part B, pink) can be optionally installed in one of the immersion tubes in order to improve the damping behavior (if unsatisfactory) of the main bushing element.

Particular attention was given to the design of the suspension elements - the coil spring and the rubber bushing. To achieve consistent behavior of the suspension over a wide range of excitation frequencies - stimulated e.g. by road irregularities, the effect of the dynamic stiffening of the rubber has to be minimized, see e.g. (Mitschke and Wallentowitz, 2014). The parallel arrangement of the bushing and the springs enables shifting of the high portion of stiffness $c_{a w}$ onto the coil spring, thus holding over $90 \%$ of the total value. The remaining stiffness realized by the bushing is then relatively low, additionally resulting in low damping $d_{a w}$ and reasonably compromised dynamic stiffening.

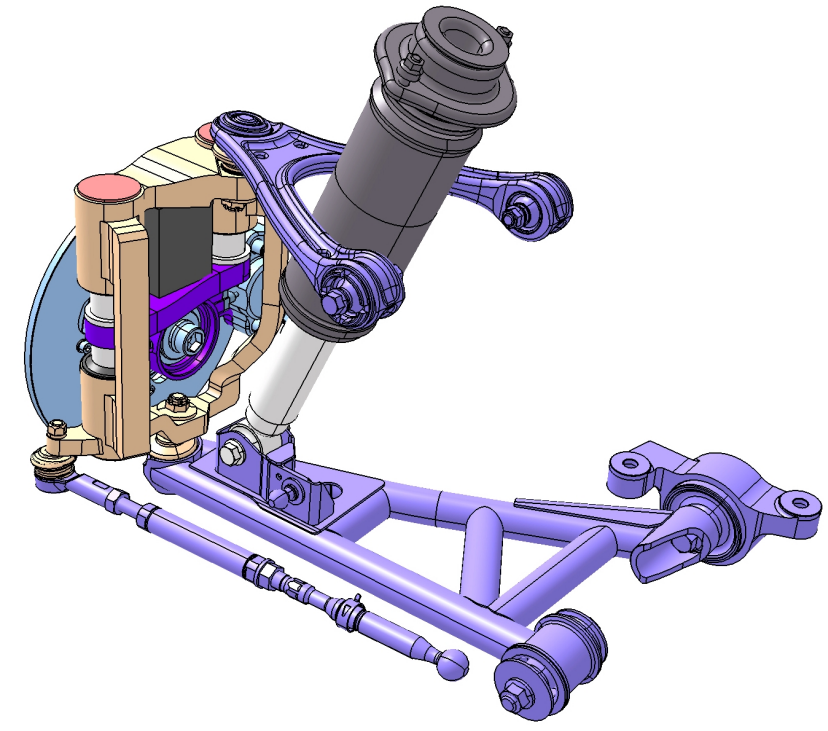

Figure 4. Technical solution of the split wheel carrier with sliding bearings in the context of a front right double wishbone suspension.

\section{Modelica Model}

To facilitate a simulative assessment the technical solution of the suspension presented in the previous section was modeled using Modelica. Using such a model, analyses on a multi-body quarter car model were performed, see Sections 5.1 and 5.2. Additionally, the suspensions were used within a total vehicle model for vehicle dynamics and driving comfort assessment, as documented in Sections 5.2 and 5.3.

In order to promote easy interoperability with the various existing automotive Modelica libraries, the created Modelica package containing all the models was consequently based upon the VehicleInterfaces base classes, see (Dempsey et al., 2006). The VehicleInterfaces library focuses on standardizing the assemblies interface definitions without presupposing a standard vehicle model architecture. Hence, the same assembly models can be reused in different model architectures.

The idea of template assembly models and parametrized models was introduced as also utilized in the PowerTrain library from DLR, see e.g. (Schweiger et al., 2005). Various parametrized models of realistic assemblies are thus inherited from template models which reflect different structure and level of model's detail. This facilitates redeclaration of the particular model within the overall vehicle models or virtual test rigs depending on the simulation purpose to be fulfilled.

The majority of virtual test rigs and maneuver scenarios as well as a couple of components and template models needed to model and evaluate the suspension concept had already been predefined within a DLR proprietary Modelica library for vehicle dynamics.

The multi-body models of a) the conventional two mass reference suspension (see model structure depicted in Fig- 


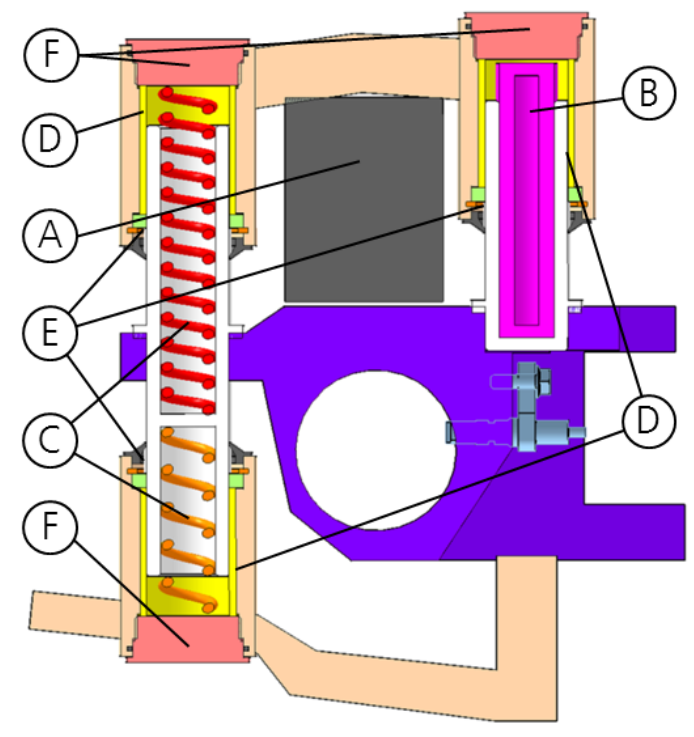

Figure 5. Cross-section view of the front right wheel carrier and components.

Table 1. Relevant masses of reference vehicle (2M) and of vehicle with proposed suspension solution (3M).

\begin{tabular}{lcc}
\hline & \multicolumn{2}{c}{ Value $[\mathrm{kg}]$} \\
Parameter & $2 M$ & $3 M$ \\
\hline Total weight incl. driver of $75 \mathrm{~kg}$ & 850 & 872 \\
Front sprung mass $m_{b}$ & 191 & 189 \\
Front unsprung mass $m_{w}$ & 26 & 23 \\
Front add. unsprung mass $m_{a}$ & N.A. & 11 \\
Rear sprung mass $m_{b}$ & 178 & 175 \\
Rear unsprung mass $m_{w}$ & 29 & 23 \\
Rear add. unsprung mass $m_{a}$ & N.A. & 15 \\
\hline
\end{tabular}

ure 6) and b) the proposed split wheel carrier design (its structure is shown in Figure 7) incorporate all relevant mass parameters as well as the nonlinearity of the force elements. In both cases the models are parameterized according to the considered small LEV. The particular masses are listed in Table 1 for the conventional reference vehicle in comparison with the vehicle with split wheel carrier. A multi-body model of the latter is visualized in Figure 8.

For elastic wishbone mounts, a simplification was adopted in that the orthogonal deformations depend proportionally only on the corresponding action forces, thus

$$
\left[\begin{array}{l}
\delta_{x} \\
\delta_{y} \\
\delta_{z}
\end{array}\right]=\left[\begin{array}{l}
f_{x} / c_{x} \\
f_{y} / c_{y} \\
f_{z} / c_{z}
\end{array}\right],
$$

and, correspondingly, for rotations. Such a mounting was additionally used to connect the toe control link of the rear suspension. For simplicity, the main bushing mounted between the wheel hub and the wheel carrier was modelled

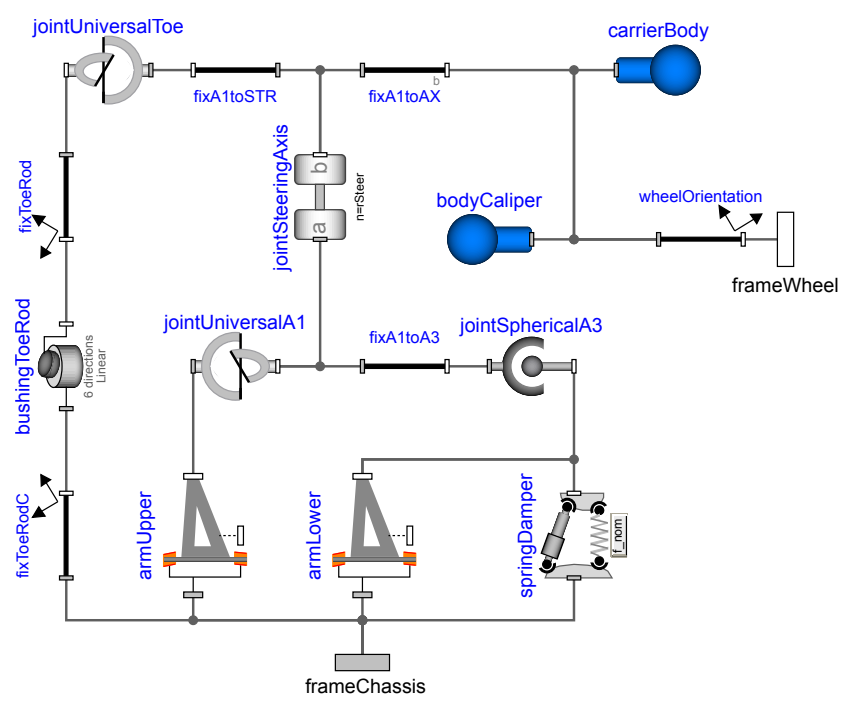

Figure 6. Modelica structure of the conventional rear suspension.

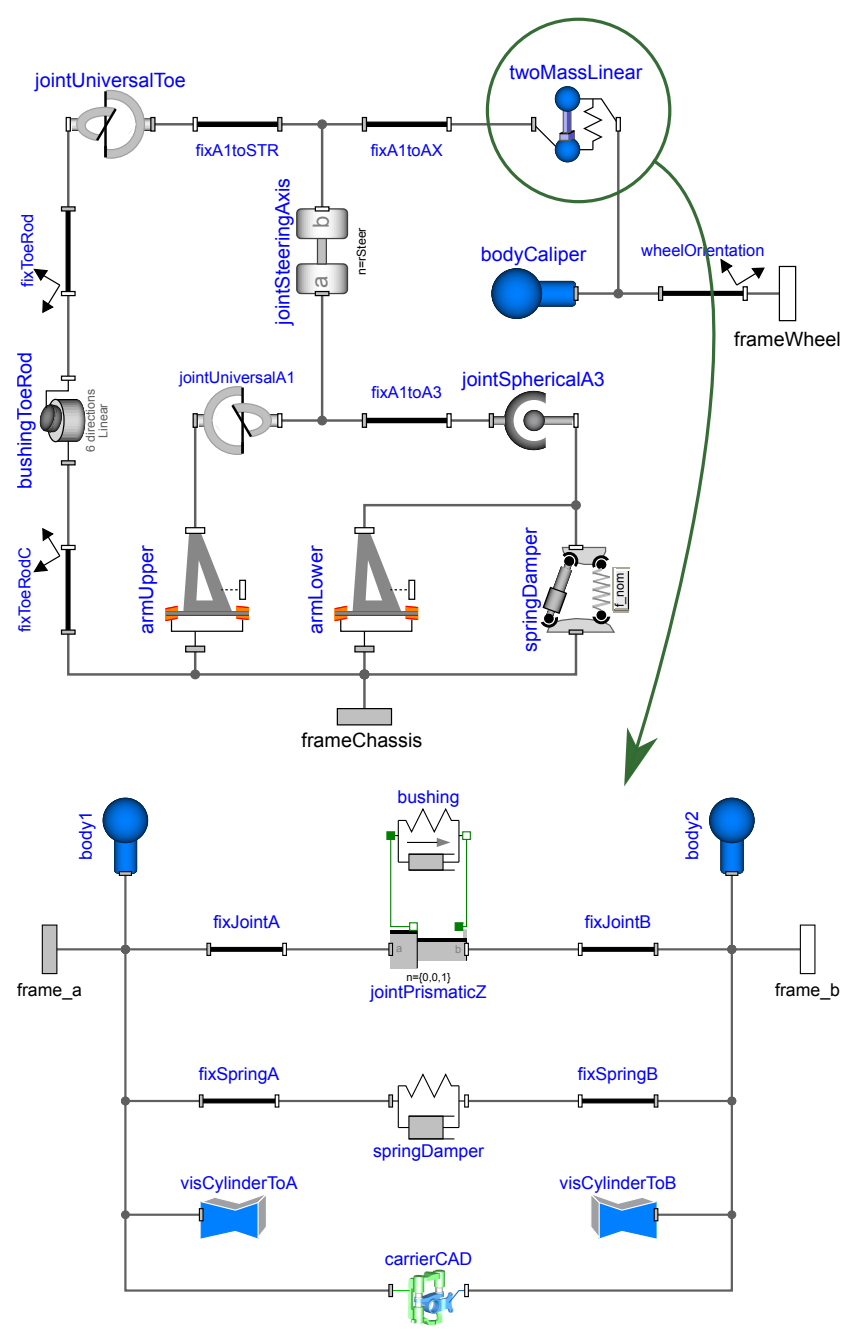

Figure 7. Modelica structure of the split wheel carrier rear suspension (above) and the split carrier submodel (below). 


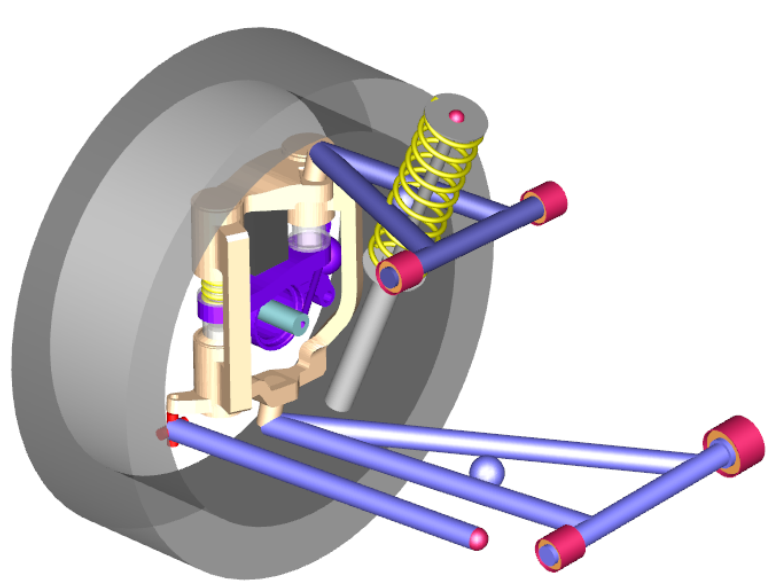

Figure 8. Visualization of the multi-body model of presented front suspension with split wheel carrier.

on the same principle. This is reasonable for low dynamic stiffening as explained in the previous section.

The vehicle body was modeled as a rigid structure with an extra mass of $75 \mathrm{~kg}$ at the location of a driver's hip joint. A linear spring-damper element was used to represent the vertical force/deflection of the tire. Additionally, the horizontal tire forces were modeled using the Pacejka Magic Formula (Pacejka, 2002) to assess the vehicle dynamics behavior.

In the following, the model of the vehicle of the two mass configuration and parametrization (2M in Table 1$)$ is called reference vehicle or simply reference.

\section{Simulation Results}

For the proposed split wheel carrier suspension the ride comfort and tire/road contact were assessed simulating both the quarter car and the full vehicle model. Additionally, vehicle dynamics were evaluated utilizing the full vehicle model only.

\subsection{Verification of the Technical Solution}

For the first verification of the eligibility of the technical design, the frequency response analysis according to (Bünte, 2011) was performed on the nonlinear quarter car multi-body models with a) the reference suspension and $b$ ) the proposed one. The vertical excitation from $0.5 \mathrm{~Hz}$ to $30 \mathrm{~Hz}$ was considered, together with the frequency-dependent decrease of the amplitude in order to reproduce the amplitude progression of the road class D from (ISO 8608). Thus, the time excitation conditions were similar to those applied for linear system analysis depicted in Figures 2 and 3.

The comparison of time domain simulation results is done in Figure 9. The multi-body models prove the reduction of the second resonance peak by the three-mass system up to frequencies about $20 \mathrm{~Hz}$. Above this frequency, the third resonance peak of the proposed suspension becomes significant which increases the response amplitude

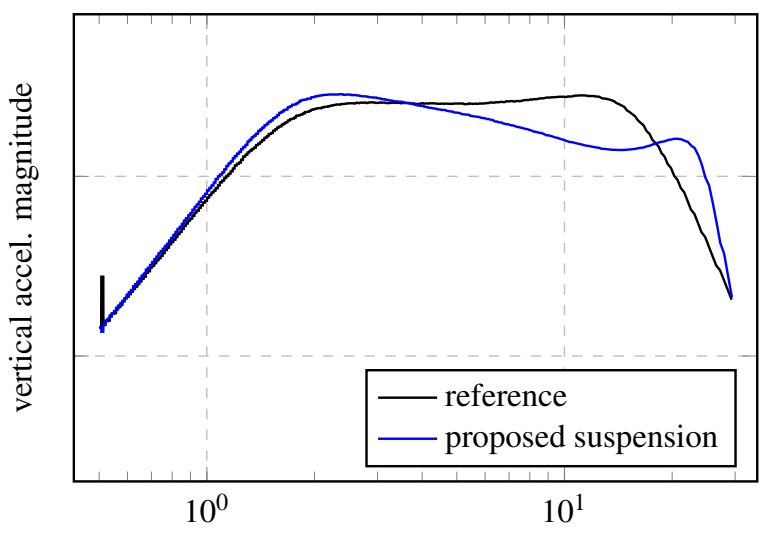

Figure 9. Frequency response of nonlinear quarter-car multibody models.

compared to the reference vehicle. In summary, this proof of concept confirmed the preliminary assessment as discussed in Section 2.

\subsection{Comfort Assessment}

For ride comfort and tire/road contact assessment, a time domain simulation of the vehicle placed on a virtual four post rig was performed. The post excitation conforms to the road class D according to (ISO 8608) driven at constant velocity of $70 \mathrm{~km} / \mathrm{h}$. The road irregularities are generated by means of a colored noise signal matching a given power spectral density (PSD). For the generation of such a signal in Modelica, the AdvancedNoise library (Klöckner et al., 2015) was utilized

For the full vehicle model, the vertical excitation signals for the left and right track were generated independent of each other, i.e. using uncorrelated signals. The excitation signals for the rear wheels were delayed by the ratio of wheel base and speed against the respective front wheels running ahead. The excitation signal together with its PSD are shown in Figure 10.

The generation of the excitation using a noise generator from the AdvancedNoise library has the advantage of a high quality stochastic signal. In contrast, this way of signal generation is computationally expensive and slows down simulation speed. Using DASSL as numerical solver on a quad-core personal computer the full vehicle simulation on average runs 50 times slower than real time. Therefore, we restricted the simulation time for the full vehicle simulation to $100 \mathrm{~s}$ which corresponds to a track length of $1944 \mathrm{~m}$. For the less expensive quarter car simulations, the simulation time of $200 \mathrm{~s}$ was applied in order to reach a more significant assessment.

The evaluation with the quarter car model used the following criteria:

- $R M S_{f z}$ : root mean square of vertical tire load,

- $R M S_{a z}$ : root mean square of body vertical acceleration, 

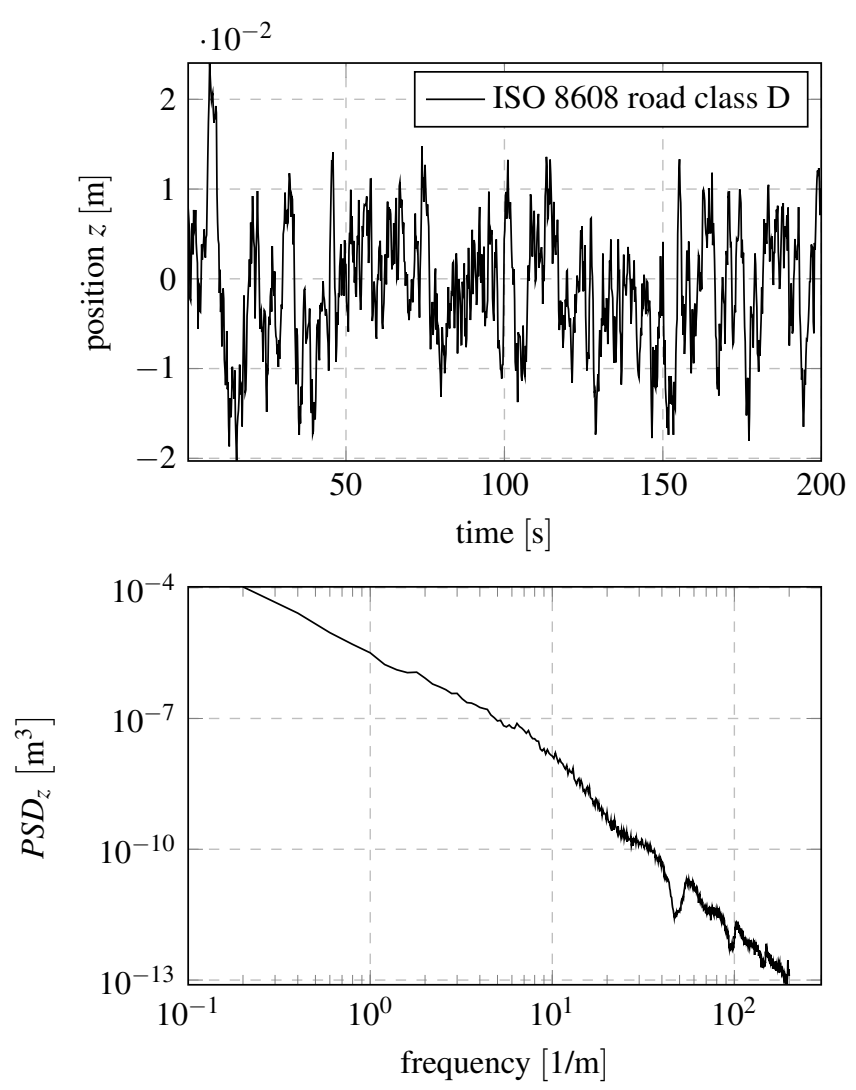

Figure 10. Exemplary road excitation signal used for vertical displacement of virtual post rig (above) and its power spectral density (PSD, below).

- $K_{\text {ges } 1}$ combined assessment criterion according to (Hennecke, 1995) for the time window of $10 \mathrm{~s}$ and

- $K_{\text {ges } 2}$ similar to $K_{\text {ges } 1}$ but for the total time of measurement.

For the full vehicle model, the following criteria were additionally evaluated:

- $R M S_{\text {pitch }}$ : root mean square of body pitch angular acceleration,

- $R M S_{\text {roll }}$ : root mean square of body roll angular acceleration.

The collectivity of criteria evaluated by simulations is shown in Table 2 and Table 3.

Supplementary to these concluding values, Figure 11 depicts the signals of the combined comfort assessment criteria $K_{g e s 1}$ and $K_{g e s 2}$ from simulations on the virtual quarter car test rig. These criteria show the improvement for the proposed suspension (plotted in red) against the reference vehicle (blue). For tire/road contact, the improvement of about $17 \%$ is reached - as indicated by $R M S_{f z}$ in Table 2. This trend is even more evident for the full vehicle simulation, see Table 3 . Here, the comfort improvement is about $25 \%$ and the road contact improvement is up to about $38 \%$. These results are achieved by
Table 2. Resulting comfort assessment criteria values for quarter car simulations with a simulation time of $200 \mathrm{~s}$.

\begin{tabular}{lcccc}
\hline Criteria & Unit & $\begin{array}{c}\text { Refe- } \\
\text { rence }\end{array}$ & $\begin{array}{c}\text { Proposed } \\
\text { suspension }\end{array}$ & $\begin{array}{c}\text { Difference } \\
{[\%]}\end{array}$ \\
\hline$R M S_{f z}$ & $\mathrm{~N}$ & 112.50 & 93.30 & -17.07 \\
$R M S_{a z}$ & $\mathrm{~m} / \mathrm{s}^{2}$ & 0.47 & 0.43 & -7.89 \\
$K_{\text {ges } 1}$ & - & 9.13 & 8.50 & -6.96 \\
$K_{\text {ges } 2}$ & - & 8.59 & 7.97 & -7.24 \\
\hline
\end{tabular}

Table 3. Resulting comfort assessment criteria values for full vehicle simulations with a simulation time of $100 \mathrm{~s}$.

\begin{tabular}{lcccc}
\hline Criteria & Unit & $\begin{array}{c}\text { Refe- } \\
\text { rence }\end{array}$ & $\begin{array}{c}\text { Proposed } \\
\text { suspension }\end{array}$ & $\begin{array}{c}\text { Difference } \\
{[\%]}\end{array}$ \\
\hline$R M S_{f z}$ & $\mathrm{~N}$ & 702.9 & 433.2 & -38.37 \\
$R M S_{a z}$ & $\mathrm{~m} / \mathrm{s}^{2}$ & 1.450 & 0.963 & -33.59 \\
$R M S_{\text {pitch }}$ & $\mathrm{rad} / \mathrm{s}^{2}$ & 2.005 & 1.430 & -28.68 \\
$R M S_{\text {roll }}$ & $\mathrm{rad} / \mathrm{s}^{2}$ & 4.707 & 3.462 & -26.45 \\
$K_{\text {ges } 1}$ & - & 32.670 & 24.412 & -25.28 \\
$K_{\text {ges } 2}$ & - & 34.260 & 26.135 & -23.72 \\
\hline
\end{tabular}

virtue of the proposed split wheel carrier system while using a preliminary parametrization.

\subsection{Vehicle Dynamics Assessment}

To achieve an evaluation of the proposed suspension concept also in terms of vehicle dynamics, two standard driving maneuvers were simulated: a) quasi steady state cornering on a circle with a radius of $40 \mathrm{~m}$ while slowly increasing the vehicle speed and b) steering angle sine sweep at a constant speed of $80 \mathrm{~km} / \mathrm{h}$. For a) the necessary steering wheel angle $\delta_{H}$ to keep the vehicle on the circle was recorded and the resulting self-steering gradient SSG was computed. During b) the magnitude $a_{y, \text { gain }}$ and phase angle $a_{y, \text { phase }}$ of the lateral acceleration frequency response were recorded and assessed.

The evaluation of the recorded criteria of both vehicles indicated just negligible difference for both maneuvers, i.e. the suggested technical solution of the proposed suspension has negligible influence on the lateral vehicle dynamics. This is particularly reached due to the sufficient stiffness of the mechanism when exposed to lateral and longitudinal forces and torques. Consequently, only marginal changes in the wheel attitude can be observed in most cases, as demonstrated in some extent in Figure 12 for a toe angle change under lateral force load, and in Figure 13 for a track change under variable vertical load. Both Figures result from the suspension elastokinematics analysis.

Concluding from the two simulated maneuvers, the vehicle equipped with the proposed suspension appears to have also a good driving behavior. 

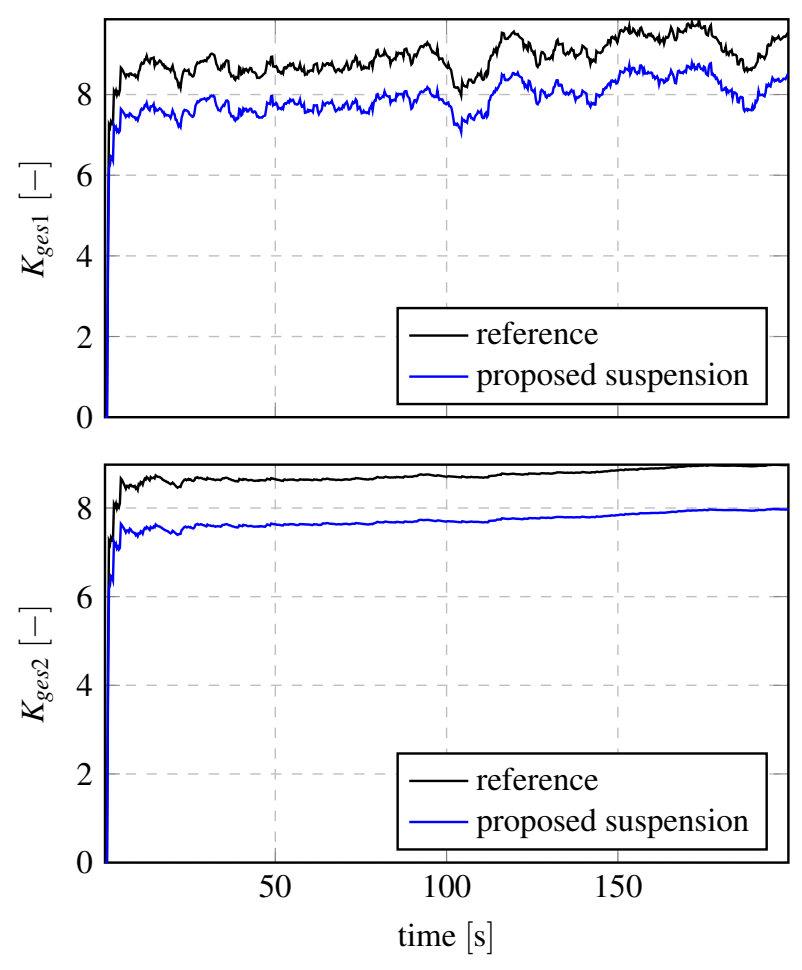

Figure 11. Comfort assessment criteria signals from quarter car simulations: $K_{g e s 1}$ for time window of $10 \mathrm{~s}$ above and $K_{g e s}$ for the total simulation time (200 s) below.

\section{Conclusions}

A suspension design solution was proposed to compensate for unfavorable effects on ride comfort and tire/road contact in the context of light-weight electric vehicles. The suggested mechanical design introduces the separation of the wheel hub from the wheel carrier allowing for a vertical relative movement between both parts by means of a prismatic joint. The realization utilizes two linear sliding bearings which house the auxiliary suspension elements a design resembling a motorcycle front fork.

A comprehensive investigation on the influence of such suspension design on the ride comfort and tire/road contact was done. Simulation results show that the ride comfort can be improved significantly while there is negligible influence on the vehicle dynamics. The relative deflection in the prismatic joint introduced into the split wheel carrier is bounded to a few millimeters when the system is operated under common driving conditions.

\section{Acknowledgements}

The authors would like to thank Mr. Uwe Bleck for sharing his expertise on vehicle suspensions and vehicle dynamics.

\section{References}

T. Bünte. Recording of model frequency responses and describing functions in modelica. In The 8th International Mod-

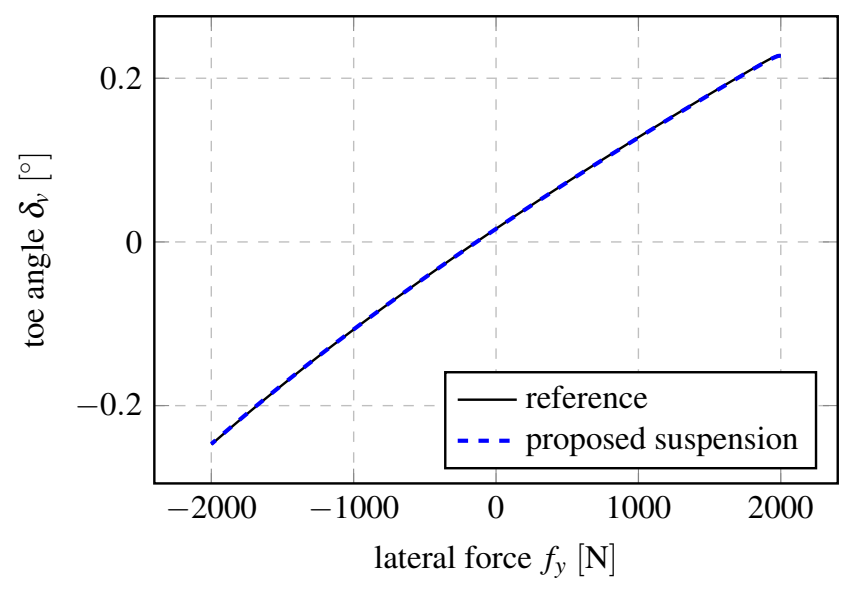

Figure 12. Change of the wheel toe angle of rear suspension under lateral load.

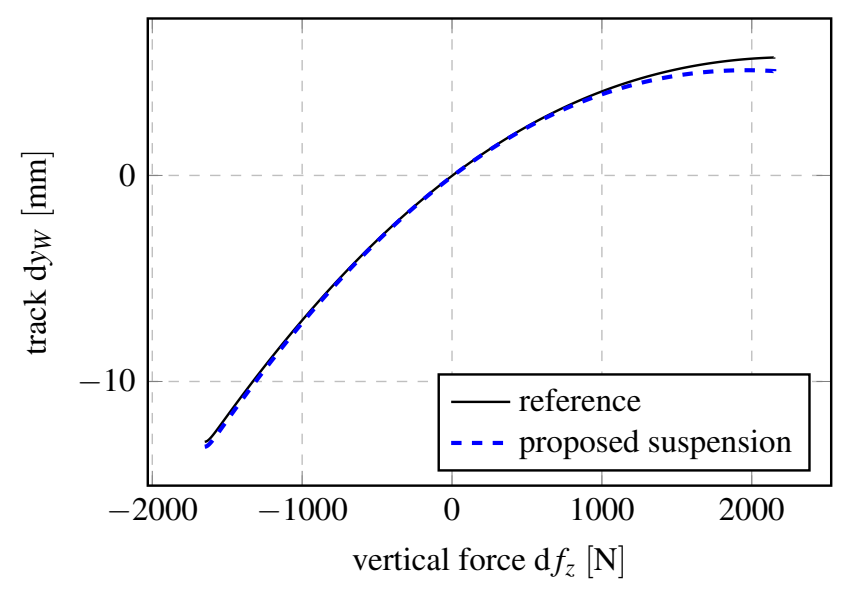

Figure 13. Change of the wheel track of rear suspension under vertical load change.

elica Conference, Dresden, Germany, 2011. URL http: //elib.dlr.de/68920/.

M. Dempsey, M. Gäfvert, P. Harman, Ch. Kral, M. Otter, and Treffinger P. Coordinated automotive libraries for vehicle system modelling. In The 5th International Modelica Conference, Vienna, Austria, 2006.

J. K. Hedrick and T. Butsuen. Invariant properties of automotive suspensions. Journal of Automobile Engineering, pages 2127, 1990.

D. Hennecke. On the Assessment of the Riding Comfort of Passenger Cars under Transient Excitation. PhD thesis, TU Braunschweig, Düsseldorf: VDI Verlag, 1995. In German.

ISO 8608. Mechanical vibration - road surface profiles - reporting of measured data, 1995.

A. Klöckner, A. Knoblach, and A. Heckmann. How to shape noise spectra for continuous system simulation. In The 11th International Modelica Conference, pages 411-418, Paris, France, 2015. Linköping University Electronic Press, 
Linköpings Universitet. URL http://elib.dlr.de/ $98408 /$.

M. Mitschke and H. Wallentowitz. Dynamics of Motor Vehicles. Springer Vieweg, 5 edition, 2014. ISBN 978-3-658-05068-9. In German.

H. B. Pacejka. Tyre and Vehicle Dynamics. Elsevier Ltd, Oxford, 2002.

D. Ryba. Improvements in dynamic characteristics of automobile suspension systems part 1: Two-mass systems. Vehicle System Dynamics, pages 17-46, 1974a.

D. Ryba. Improvements in dynamic characteristics of automobile suspension systems part 2: Three-mass systems. Vehicle System Dynamics, pages 55-98, 1974 b.

S. M. Savaresi, C. Poussot-Vassal, C. Spelta, O. Sename, and L. Dugard. Semi-active suspension control design for vehicles. Butterworth-Heinemann/Elsevier, 2010.

Ch. Schweiger, M. Dempsey, and M. Otter. The PowerTrain Library: New Concepts and New Fields of Application. In The 4th International Modelica Conference, Hamburg-Harburg, Germany, 2005.

J. Stoffregen. Motorcycle technology. Springer Vieweg, 2012. ISBN 978-3-8348-1716-7. In German, DOI 10.1007/978-38348-2180-5. 